\title{
An optimization algorithm for a capacitated vehicle routing problem with time windows
}

\author{
PINAR KIRCI \\ Engineering Sciences Department, Istanbul University, Istanbul, Turkey \\ e-mail: pkirci@istanbul.edu.tr
}

MS received 15 February 2016; revised 20 March 2016; accepted 1 April 2016

\begin{abstract}
In this paper, vehicle routing problem (VRP) with time windows and real world constraints are considered as a real-world application on google maps. Also, tabu search is used and Hopfield neural networks is utilized. Basic constraints consist of customer demands, time windows, vehicle speed, vehicle capacity and working hours. Recently, cost and on-time delivery are the most important factors in logistics. Thus, the logistic applications attract attention of companies. In logistic management, determining the locations of delivery points and deciding the path are the vital components that should be considered. Deciding the paths of vehicles provides companies to use their vehicles efficiently. And with utilizing optimized paths, big amounts of cost and time savings will be gained. The main aim of the work is providing the best path according to the needs of the customers, minimizing the costs with utilizing the VRP and presenting an application for companies that need logistic management. To compare the results, simulated annealing is used on special scenarios. And t-test is performed in the study for the visited path in $\mathrm{km}$ with p-value of 0.05 .
\end{abstract}

Keywords. Vehicle routing problem; google maps; tabu search; hopfield neural networks; simulated annealing.

\section{Introduction}

In ever evolving and improving world, logistics becomes a remarkable factor between competing companies. The companies present many innovations about logistics area to be able to deliver, provide the needed products and service to their customers on time, at the demanded place. Latest developments in technology induce the improvements about logistics area in companies. It is very important and vital to provide the demands of the customers on time for the companies' prestige. Besides, while providing the needs of customers, to provide better working platforms for their own staff together with having maximum profit and minimum cost are the main aims of the companies.

Hence, companies focus on explorations about vehicle routing problem (VRP). Researches about VRP started with travelling salesman problem (TSP) [1]. The VRP problem consists of the problems that reveal during the delivery/collection of products and presenting of service to the customers from one or more depot. The VRP varies according to its application areas, thus many different solutions, methods are presented for them. The solutions mostly focus on the efficient utilization of definite amounts of capacity having delivery vehicles with considering vehicle capacities and service time limits of the customers [2].
An extension of the bacterial foraging optimization (BFO) algorithm with time-varying chemotaxis step length and comprehensive learning strategy is proposed in [3]. The adaptive comprehensive learning bacterial foraging optimization (ALCBFO) method was used on vehicle routing problem with time windows (VRPTW). An agent-based cooperative population learning algorithm for the vehicle routing problem with time windows is presented in [4]. Also, an adaptive large neighbourhood search (ALNS) heuristic algorithm was presented in [5] for the pickup and delivery problem with time windows and scheduled lines (PDPTW-SL). The capacitated vehicle routing problem (CVRP) is explored in [6]. Four algorithms are utilized to solve the considered problem: an improved intelligent water drops (IIWD) algorithm as a new swarm-based nature inspired optimization one; an advanced cuckoo search (ACS) algorithm; and two effective presented hybrid metaheuristics incorporating these methods, known as local search hybrid algorithm (LSHA) and post-optimization hybrid algorithm (POHA). A new variant of the time-dependent VRP was studied in [7] with allowing more than one edge between the nodes. And the problem was designed as the time-dependent vehicle routing problem in multigraph which derived the FIFO property. And also, a heuristic tabu search (TS) algorithm was utilized for resolving the problem. Open vehicle routing problem with 
cross-docking (OVRPCD) is addressed with presenting a simulated annealing (SA) algorithm in [8]. The SA algorithm includes many neighbourhood structures for increasing the performance on solving OVRPCD. The clustered CVRP is studied in [9]. To solve the clustered CVRP, a two-level solution approach is presented to gain high quality solutions. A metaheuristic based on iterated local search was improved for the multi-depot vehicle routing problem with simultaneous deliveries and pickups (MDVRPSDP) in [10]. Also, an adaptive neighbourhood decision mechanism was inserted into the improvement steps and then, the perturbation steps of iterated local search.

Most of the existing papers for solving VRP problem include only graphical illustrations to address the problem, but for a real life solution, map applications are required. Recently, a few papers considered to study VRP on real map whereas adding this feature will provide the VRP problem to be solved as a real life problem with working on real transmission roads and will minimize total cost of the problem.

Although the VRP with deliveries have frequently studied in real life scenarios of logistics, working on a real world application on google maps has received less attention so far. In literature, a few number of papers have used metaheuristics to solve the vehicle routing problem on google maps.

In this paper, we study the VRP problem on both real map and graph illustrations to present more significant solutions. Furthermore, we consider not only optimization of a VRP but also present a user friendly logistics application together with a database that can be utilized in logistics firms. Because, there are a few papers that include logistics applications. Also, we focus on tabu search because of its simple and flexible structure that provides better computation time. Tabu search is used for VRP and compared with SA on special scenarios.

The main aim of the paper is to present an application that optimizes the routes with considering the VRP limits. The application is developed as windows form application. The map is constructed by utilizing gmap.net library. For the related data, Microsoft SQL server is used. In the paper, the vehicles use one depot, visit delivery points at most one time and deliver the packets to the customers in between the defined time periods which are known as time windows. During the process, the minimum cost having path is produced by Tabu search algorithm. The routes are illustrated and shown as a graph on windows form and also on google maps. As a result of the optimization process, the gained outcomes are presented with graphics.

The rest of this paper is organized as follows. In section 2, VRP problem is proposed with its basic components, general aims, basic types and solution methods. Section 3 briefly presents main components of tabu search algorithm. The proposed system is given in section 4 . The presented scenarios and the gained results are compared and presented in section 5. The comparisons are given with an application of tabu search on Hopfield neural networks and SA in section 6. The paper ends in conclusion by proposing concluding remarks.

\section{VRP problem}

\subsection{Basic components of VRP}

VRP owns some components from real life: route, customers, vehicles, depot centers, drivers, constraints and aims. Route network is represented with a graph in VRP. In a graph, there are nodes and edges. In VRP, the routes are represented by edges and the customers are represented by nodes. The graphs may be directed or undirected. Customers are the units who may have a packet that should be delivered from the depot or send to a destination.

The constraints vary according to the rules of the transportation, the quality of the logistics service and the working conditions of the drivers. However, the constraints are layered in three steps. The constraints about the vehicles are as follows: vehicle capacity constraints, the sum of the spent time constraint during the delivery, the driver's daily working hours constraint that will be decided by ministry of labour and number of vehicles constraint that are waiting in a depot. The constraints about the customers are as follows: time constraint at the delivery area, the constraint of not to come by a delivered customer again. Other constraints are as follows: a vehicle may use the same route more than once, the total delivery time is more than a daily delivery time period [11].

\subsection{General aims of VRP}

In VRP, the applications are developed according to the requested aims. These aims are to minimize the delivery and vehicle costs, to optimize the number of vehicles and drivers, to optimize the spent time during the delivery period, to minimize the total visited distance. The applications may be developed according to one of these presented aims or some of these presented aims by combining them and also considering their contradictions. Then the VRP problem turns into a multi-objective decision problem.

VRP considers providing convenient service distribution for customer demands between the defined points on the map. Application areas develop according to the demands of customers. Basic application fields are fuel and diesel delivery, the arrangement of the soldiers in a battlefield, flights of airplanes, delivery of food and beverage to the restaurants, delivery of money to the bank ATMs and cash machines, student and worker services, delivery of packets that are purchased by shopping over internet, garbage collecting and transporting [12]. 


\subsection{The types of VRP and solution methods}

To develop applications in VRP, its limitations are considered. VRP is sublayered according to these limitations. VRP with time windows: every customer has time limitations, thus the delivery to the customers should be performed in between the pre-defined time periods. VRP with backhauls: the delivered packets to the customers might need to return for some reasons. The deposit of the delivered product may need to be collected, the package and wrapping of the products can be collected for recycling or the product may return by the customer. The VRP is performed together with considering these parameters. Multiple capacitated VRP: The utilized vehicles have different transportation capacities. Split delivery VRP: more than one vehicles may serve to one customer. Multiple depot VRP: the number of depots may be more than one, according to the transportation corporation and number of customers. In this case, every one of a vehicle leaves from its depot, delivers its packets and returns its depot. And different distance VRP is considered when the arrival distance to the customer and return to the depot after delivering to the customer are different during the delivery [13, 14].

\section{Tabu search algorithm}

In VRP solution methods, tabu search algorithm belongs to meta-heuristic method under heuristic methods. Tabu algorithm is developed by [15] for proposing an optimized solution for VRP. It is inspired by human memory's working principles. The basic idea of tabu search algorithm is preventing from repetitions and prohibiting or punishing the repetition in the next step. The main aim of tabu algorithm is satisfying the deficient parts of simple hill climbing heuristic (HC). In HC, new solution candidates are compared with the old solution and the proximity between solution and result is decided. The closest solution to the result in between the new solution candidates and old solution is replaced with the old solution. And this factor may cause a vicious cycle in HC, it may be stuck between more than two neighbourhoods having same distance. The properties of the searched area or not choosing the most suitable HC may route the search that has not utilized a control mechanism to the best local points. In real life problems, there are many local bests but less global bests are found and also in some situations there is sole, thus it is inevitable to emerge a vicious cycle.

In this case, tabu search algorithm advises to use memory usage to avoid a vicious cycle. Already visited or the candidate solutions that should not be visited are kept in a short time memory like list which is called as tabu list. Thus, the steps in the related list are not used for a definite period of time. The search avoids of local searching and closer results to the real result are gained [16-18].

\subsection{Main components of tabu search algorithm}

Basic components of tabu search algorithm are a starting solution, movement mechanisms, neighbourhood, memory, tabu list, tabu destroy criterions and stopping. A starting solution is needed for the solution of tabu search algorithm. Different solution methods may be used to acquire a starting solution. These results are gained as randomly and also a solution can be gained with heuristic methods. In the project, the starting solution is gained with greedy approach. In greedy approach, the possible closest one to the solution is chosen. According to this approach, firstly a city is chosen as a starting point, then the closest another city is chosen, thus a city list is formed with adding closest city to the last one. The gaining of new solutions with the alteration over existing solutions is performed by movement mechanism. The possible movements in movement mechanism form the neighbourhood table of existing solution. These movements can arise as the movements between the routes and for the routes.

One of the most important components of tabu search algorithm is a neighbourhood structure. The main aim of optimizing the solution is to choose best movements. To get true results with the algorithm, neighbour choosing is also an important factor. While choosing the neighbours, $n+1$ neighbours should be decided for $n$ chosen points.

Another component of tabu search algorithm is memory. The arised states during the tabu search algorithm and the states that should not be searched are listed in a list which is known as tabu list. And the states that should not be allowed are named as tabu. The memory is temporary and is used to avoid a vicious cycle. After a while, these movements are removed from tabu list and they are allowed to be used [19]. Tabu list keeps the states that should not be looked at during the search. The size of the tabu list affects the result. Tabu destroy criterions are the states where the tabu disappears. Most general tabu destroy criterion is getting closer result with the produced solution than older solution, as a result of this, the tabu is destroyed. The effectiveness of tabu search algorithm increases with utilizing this criterion. Tabu search algorithm continues its search till providing one or more stopping rule. Some of these rules are: the chosen neighbour solution has no neighbour, to reach a definite number of iterations, to reach a definite value of a solution, the algorithm gets stuck and it does not produce better results [20].

\section{Proposed system}

VRP is one of the most important topics in management. Here, the problem consists of one depot and randomly distributed locations having customers' deliveries with minimum cost, minimum number of vehicles and shortest route. In addition to the basic limitations of VRP, every customer is visited once and every route starts and ends at the depot. Main 
components of VRP are composed of delivery network, customers, vehicles, depots, drivers, limitations and aims.

In the system, many basic parameters of VRP are considered: minimizing the total transportation cost by considering the travelled total distance of vehicles, minimizing the number of vehicles which are utilized to deliver the packets to the customers. And computing and minimizing the total time period of the related delivery path with considering the capacity of the delivery vehicle. These aims are intended to be provided in the presented paper. The produced results are presented together with older results of previous routes, thus user may compare the gained results with previous results. VRP with time windows and tabu search algorithm is utilized in the project.

The improved application can be utilized by cargo corporations. Routes will be defined according to the needs of the corporations. In the application, VRP with time windows will decide the routes according to the customer's time intervals. The application can be used in many different areas such as defining routes for space vehicles in space stations. The time windows will be important for space stations because the time intervals of sun is vital for space stations. And VRP with time windows can easily be modified according to the needs of space vehicles. Also, it can be used in fuel and diesel delivery, student and worker services, garbage collecting and transporting. To provide a more convenient system, the positions of the delivery points and depot are assigned with using mouse on the gmap.net library. And also the determined routes are presented over the map of gmap.net library. The coordinates of depot and customers are kept in the database. With the help of these values, the coordinates on the map are matched with the pixels on the windows forms and graphical illustrations are performed over windows forms. And .Net Visual Studio 2013 Standard edition with SQL Management Studios database is utilized.

\subsection{System interfaces}

Interfaces of the presented VRP system are formed as Windows forms with Microsoft Visual studio C\#. Main interface is designed as neat and user friendly. The main aim of the presented interface design is providing easy to use interfaces for users. Main interface is composed of two parts. The user can easily manage the program and make some processes over the first part. The routing will take place over second part which is a gmap.net map together with the illustrations of the depot and customers' locations. In addition to this, with the route button, the optimized route is illustrated over the map. With the graphic button, the route over the map is illustrated over the graph. Added depots and cargo delivery points are represented with DataGridViews. The stations interface is the first interface of the system. Depot and cargo delivery determination processes take place over stations interface.

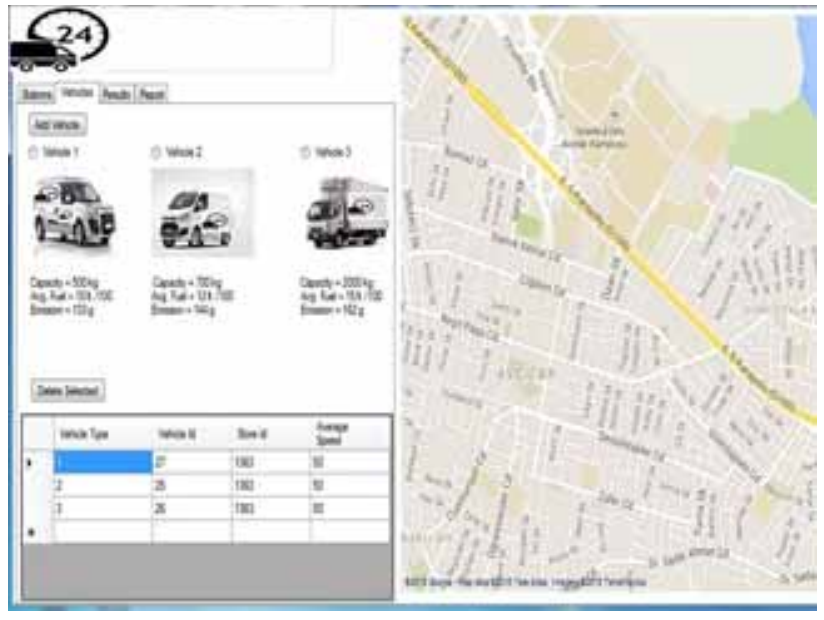

Figure 1. Main interface with vehicles part.

The vehicles are added and removed from the system over vehicles interface. Added vehicles are illustrated over DataGridView as given in figure 1. After the route defining process, according to the optimized result, the delivery order of the cargos and serving time periods to the customers are defined at report interface. Also, the cargo delivery addresses are proposed with excel. Depots' and cargo delivery points' locations can be determined and illustrated on the gmap.net map. Addresses, coordinates of related depot and cargo delivery points are gained from gmap.net map. And also processes are performed according to the information. Besides, with the route button, routes can be illustrated over the map.

The results are presented in one interface. Here, the results are given after the routing processes with DataGridView. The results are gained before and after using the presented system and they are given together with the routes. And also, these results are illustrated as graphically on an interface together with the DataGridView. The DataGridView includes whole routes and lists starting from the last one till the first one. In the DataGridView, many data are presented about routes: route no, route, the sum of the route in meters before using the proposed system, the sum of the route in meters after using the proposed system, the cost of the route in kuruş (Turkish lira) before using the proposed system, the cost of the route in kuruş (Turkish lira) after using the proposed system, the emission of the route in gr before using the proposed system, the emission of the route in gr after using the proposed system, the total spent time in minutes before using the proposed system, the total spent time in minutes after using the proposed system. For the calculations in the system, real distances are taken as km by gmap.net according to the gained distance, with using average vehicle speed and average emission rates. With the proposed system many results are gained: cost, distance, emission and spent time. These results are given with graphics by comparing the optimized solution with the initial solution. 
In the report interface, the information of addresses of customers that will be visited, the information of the cargo vehicle's visiting time to the customer and the information of time period of serving to the customer data are given. In the presented application: the corporation's working hours starts at $08.00 \mathrm{AM}$, the vehicle leaves the depot, visits whole delivery points and turns back to the depot, the service period is $15 \mathrm{~min}$ for every customer. If needed, the data on the page can be transferred to a excel file and editing can be done. After the routing process, packets' delivery data can be listed. Delivery addresses, the distances of these delivery addresses as meters and each packet's service time periods are given on the file.

\section{Presented scenarios}

After choosing the depot and cargo delivery points, the system starts with greedy algorithm and produces a starting solution. Then, with utilizing the produced starting solution, tabu search algorithm is used. And optimized solution is gained and consequently the route is produced. As shown in figures 3 and 7 the vehicle left the depot, wandered the whole delivery points according to the tabu search algorithm and turned back to the depot. To avoid the disorder, the path from the depot to first cargo delivery point is represented with blue coloured arrow and the path from the last cargo delivery point to the depot is represented with a black coloured arrow. Other paths between the cargo delivery points are represented with a green coloured arrow.

In the system, before adding a vehicle, the user should add a depot for the application. If the user does not add a depot before adding a vehicle then the system will present a warning message because the user tries to add a vehicle to an application that does not have a defined depot. To add vehicles, user should choose one of the three vehicles that are given on the page or the system may decide the vehicle according to the packet amounts in kilogram that will be delivered. The vehicles' capacity, average fuel usage rate and emission rates are given together with their pictures on the page. The user can decide which vehicle to choose according to vehicle's properties. The chosen vehicle's basic information are shown on the page in the DataGridView which are the number of the vehicle, its type, its related depot number and its average speed.

Capacity and average fuel usage rate are compared before and after tabu search algorithm which is applied to the system. Results are gained before optimizing the system and also results are gained after optimizing the system with the proposed algorithm, then results are compared. Also, the vehicles that will be used in the scenarios are decided by the program according to the cargo amounts. In the presented project, every cargo is assumed to be $100 \mathrm{~kg}$. The sum of the cargo amount is calculated and the program decides which vehicle will be used in the scenario. Added vehicles, chosen cargo positions and depots can be erased from DataGridView. The vehicles' speed limit is decided as $50 \mathrm{~km} / \mathrm{h}$. Because the vehicle speed limit is $50 \mathrm{~km} / \mathrm{h}$ in the city. It is assumed that one litre of fuel's cost is around 4.75 TL. The total cost of the whole scenarios' are computed in Turkey's currency unit (TL).

\subsection{Scenario-1: Localization of customers and the depot between the counties of Istanbul, Turkey}

In the first scenario, 16 customers are served between the counties of Bahcelievler, Zeytinburnu, Bayrampasa, Kagithane, Beyoglu, Maslak, Bahcekoy, Zekeriyakoy, Poyraz, Kaynarca, Akbaba, Gorele, Arnavutkoy, Umraniye, Kadikoy and Atasehir with the depot in Uskudar, Istanbul, Turkey as given in figures 2, 3, 4 and 5. In figure 2, the depot and customers' locations are positioned.

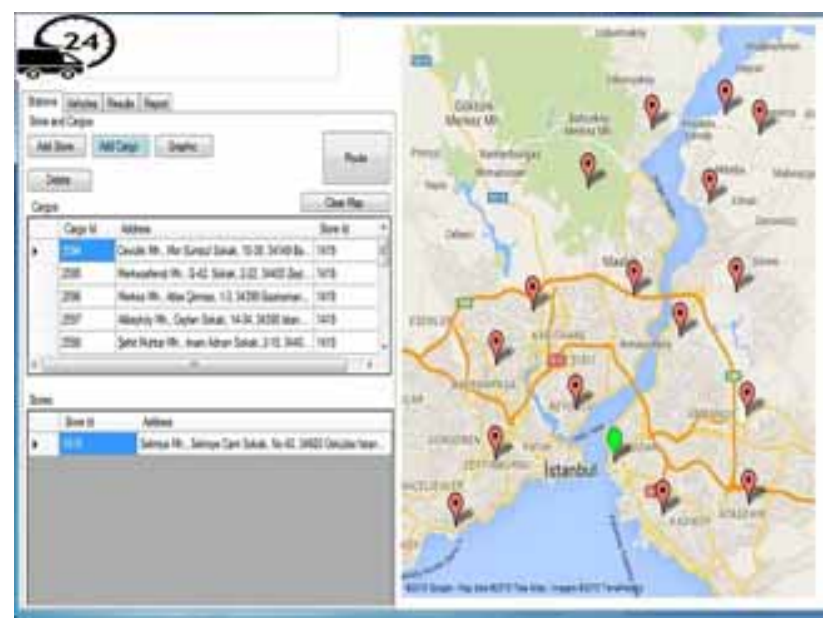

Figure 2. Localization of customers and the depot on the map.

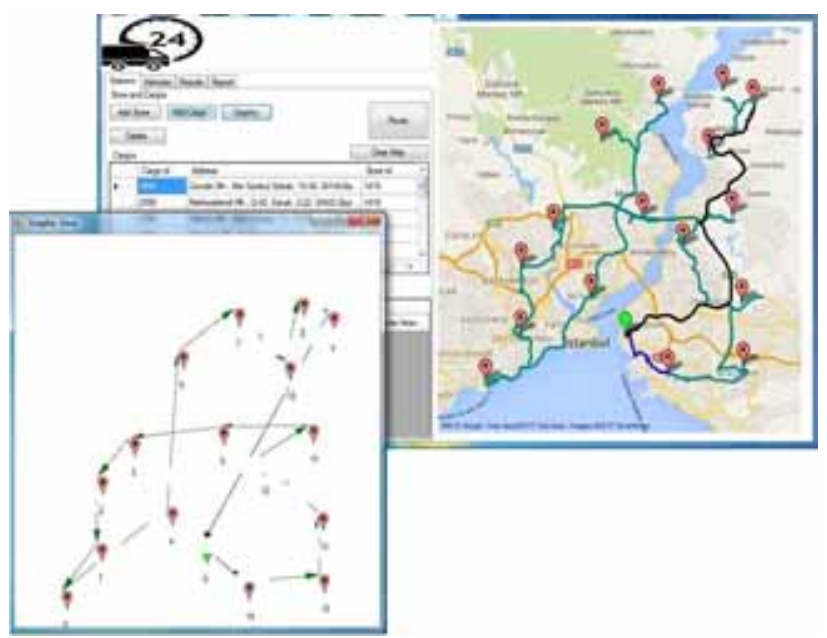

Figure 3. Shortest path between the customers and the depot over the google maps and over a graph. 


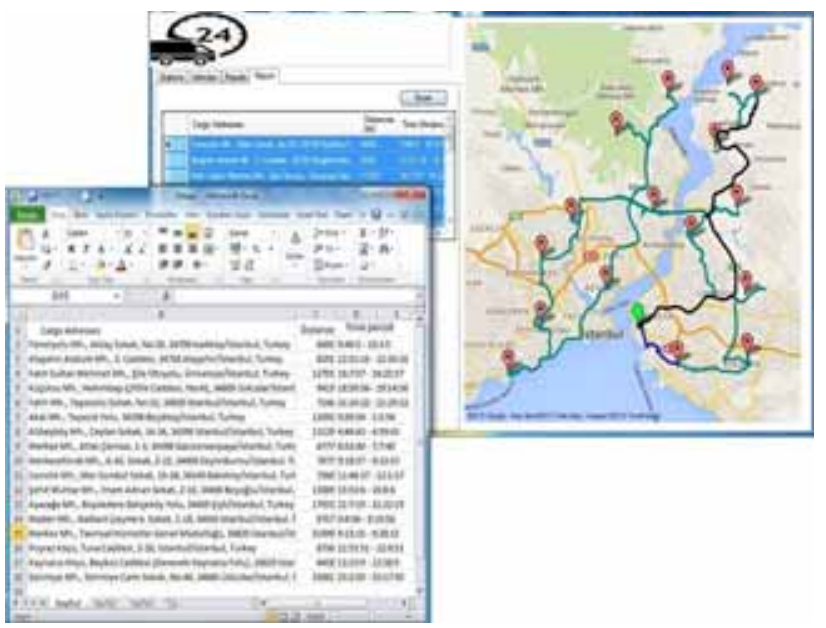

Figure 4. Detailed list of customers with their private data.

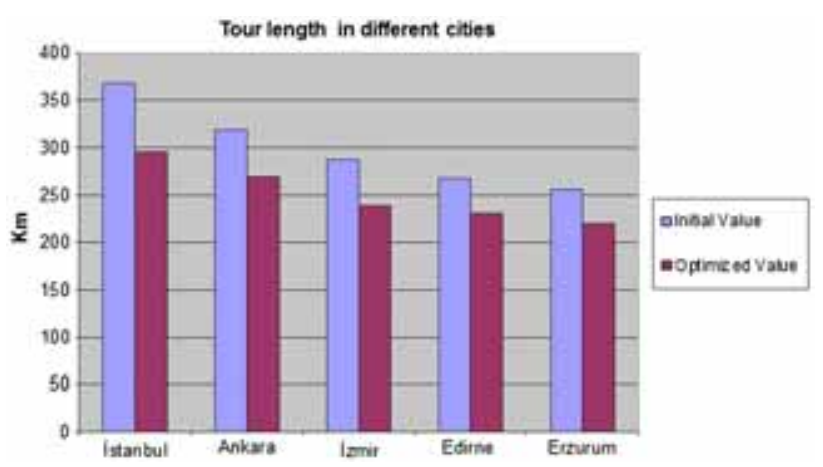

Figure 5. The tour lengths in different cities with their counties.

In figure 3 , the shortest path is computed according to the presented system. The route is illustrated over the google maps and the graph for the chosen vehicle.

In figure 4, the detailed list of customers with their private data is given. The list includes many vital data about the customers for the system. The addresses of the customers, the travelled distance between the locations, arrival time to the customers, leaving time from the customers, time windows of the customers, data of the cargo, data of the depot and data of the vehicle are kept in the list.

The tour lengths change in different cities depending on the crowded population of the city. Sixteen counties are visited in different cities on the map to reveal the tour lengths' changes depending on the crowdedness and vastness of the cities in figure 5. Because the increase in population produces bigger cities with more roads, traffic and buildings. The tour lengths are mostly affected by the traffic and one way roads of the considered city. Especially, in Istanbul and Ankara which are the biggest cities of Turkey, one way roads cause great tour length increases because of working on a real time map. Thus, Istanbul has the longest tour length when compared to other cities.

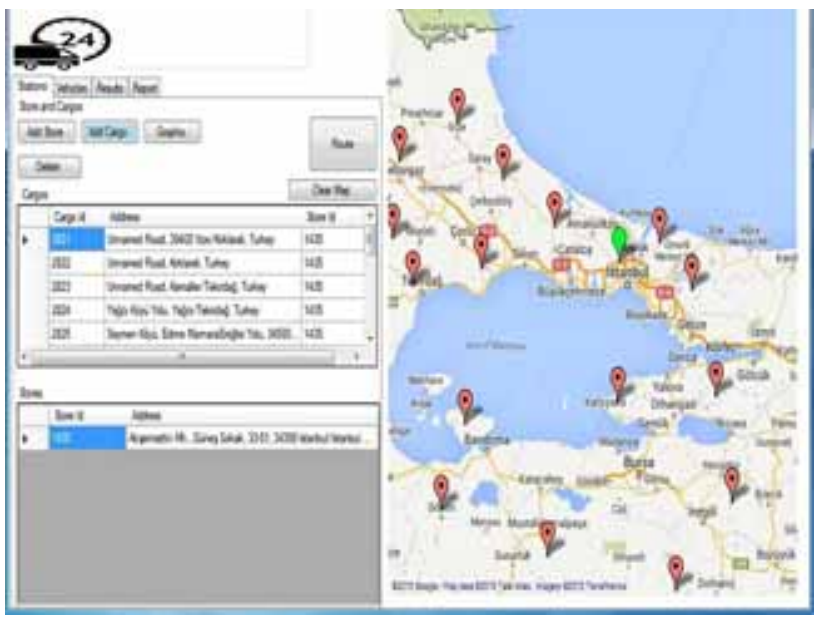

Figure 6. Localization of customers and the depot on the map.

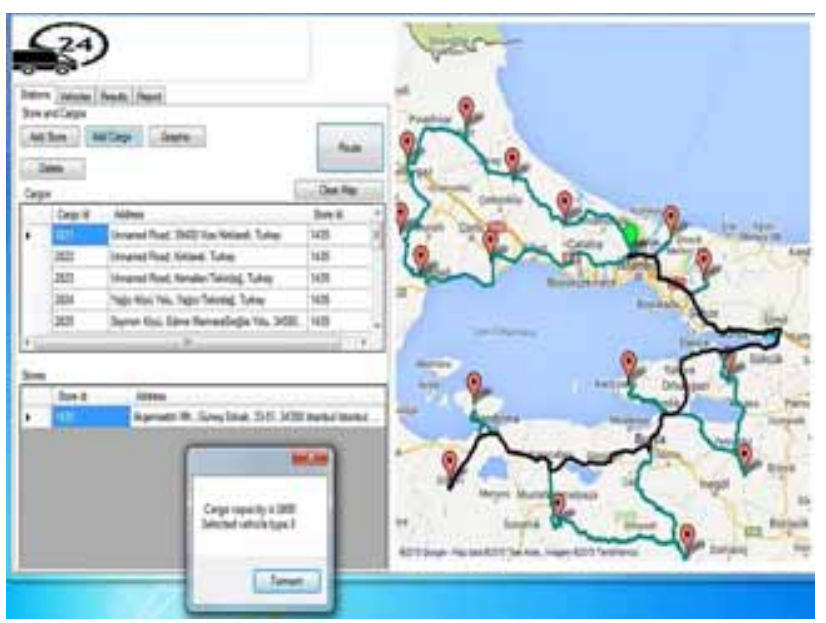

Figure 7. Shortest path between the customers and the depot over the google maps.

\subsection{Scenario-2: Localization of customers and the depot between the cities in Turkey}

In the second scenario, 16 customers are served between the cities of Tekirdag, Istanbul, Izmit, Bursa, Kırklareli, Kutahya, Balıkesir with the depot in Istanbul, Turkey as given in figures $6,7,8,9$ and 10. In figure 6 , the depot and customers' locations are positioned.

In figure 7 , the shortest path is computed according to the presented system and the route is illustrated over the google maps for the chosen vehicle.

In figure 8 , the shortest path is computed according to the presented system and the route is illustrated over a graph for the chosen vehicle.

In figure 9, the detailed list of customers with their private data is given. The list includes many vital data about the customers for the system.

The tour lengths change in different regions depending on the surface area of the region. Sixteen cities are visited 


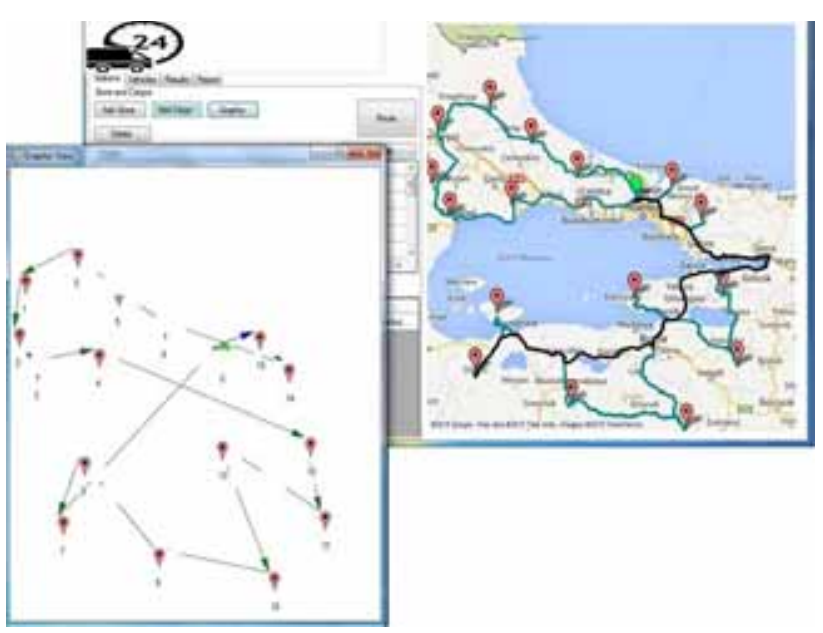

Figure 8. Shortest path between the customers and the depot over a graph.

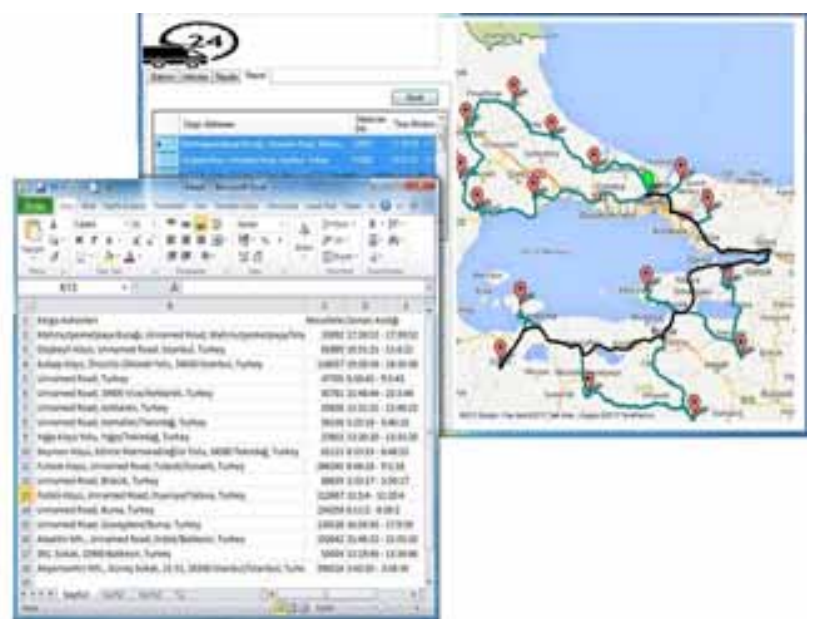

Figure 9. Detailed list of customers with their private data.

in different regions on the map to reveal the tour lengths' changes depending on the crowdedness and vastness of the regions in figure 10. Thus, biggest surface area owning region which is Central Anatoli region has the longest tour lengths when compared to other regions.

\subsection{The travelled distance and cost results of scenario-1 and scenario-2}

In figures 11 and 12, initial results (without performing the presented system) and optimized results (after performing the presented system) are given. The results of the traversed distance are given in meters $(\mathrm{m})$ in figure 11 . The total cost of the consumed fuel before applying the presented system and the total cost after applying the presented system are given in Turkey's currency unit (TL) in figure 12. Also, optimization rates are proposed on figures 11 and 12 .

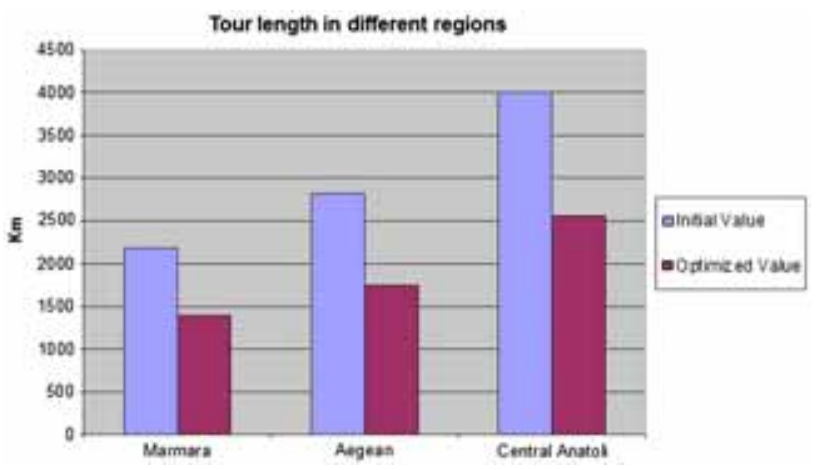

Figure 10. The tour length between cities in different regions.

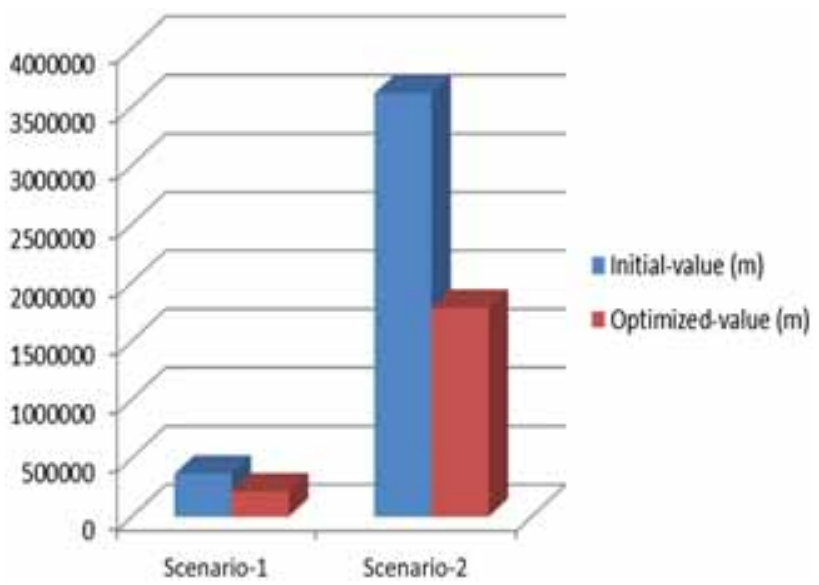

Figure 11. The travelled distance between the customers and the depot.

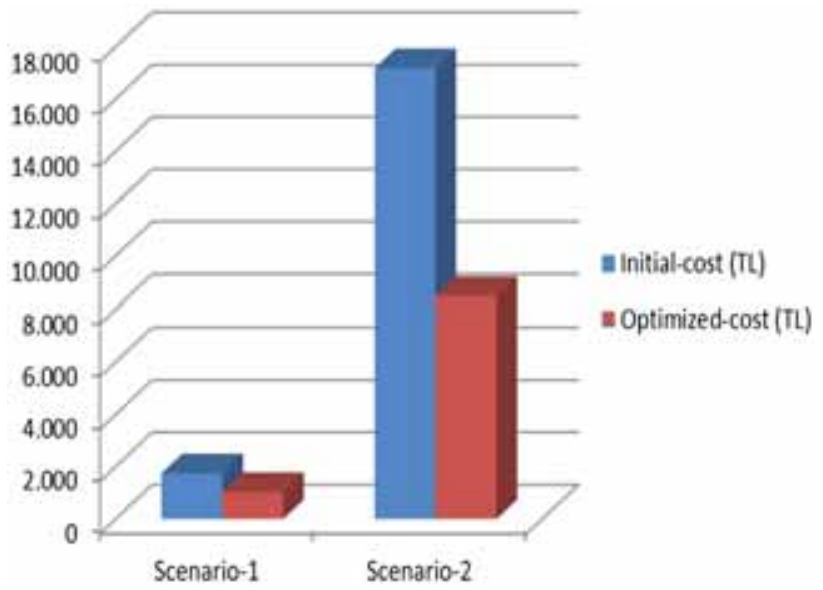

Figure 12. The total cost of the consumed fuel.

The data is examined by the paired sample t-test method. The test results are presented in table 1. Gained results show that the tabu search algorithm performs significantly better as in t-test with $p$ value of 0.05 in terms of visited path in $\mathrm{km}$. 
Table 1. Statistical results of paired t-test.

\begin{tabular}{lcc}
\hline & \multicolumn{2}{c}{ Path in km } \\
\cline { 2 - 3 } & $\begin{array}{c}\text { Before optimization } \\
(\mathrm{km})\end{array}$ & $\begin{array}{c}\text { After optimization } \\
(\mathrm{km})\end{array}$ \\
\hline Sample size (n) & 20 & 20 \\
Mean (X) & 33,595 & 20,215 \\
Standard & 24,337 & 14,113 \\
$\quad$ deviation(s) & & \\
T-value (t) & $5,607,201,688$ & \\
$P$ value & $104,335 \times 10^{-5}$ & \\
\hline
\end{tabular}

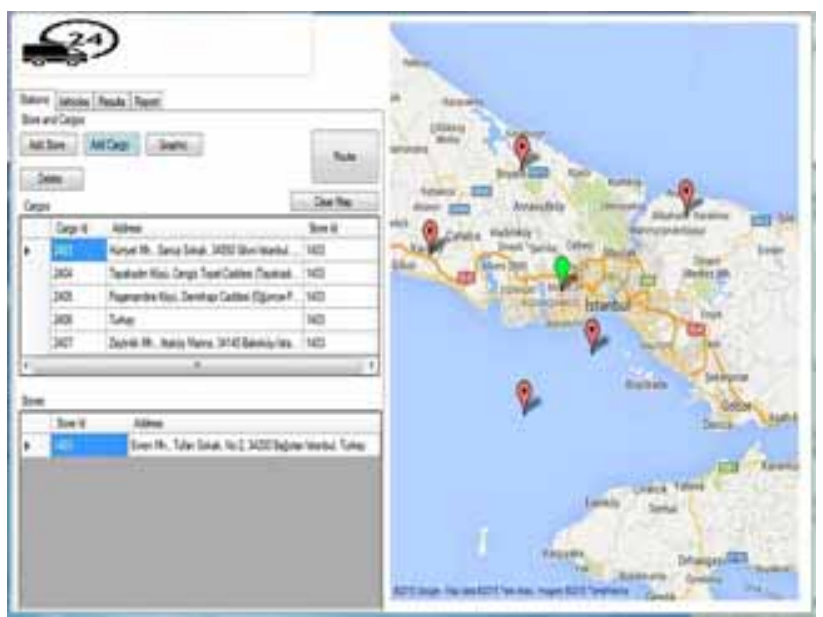

Figure 13. Localization of the customers and the depot.

\subsection{Scenario-3: Localization warning}

In the project, if a user positions a customer's location in the sea as in figure 13 , the system warns the user about the inappropriate position of the chosen location with a warning message as presented in figure 14. And the user is forced to position the customer's location on the land over the map.

\section{Comparisons with applications of tabu search}

\subsection{Hopfield neural network}

The connection between neural networks and physical systems which are considered in statistical mechanics is established in [21] by Hopfield. Real world situation problems are easily constructed as optimization problems. And the Hopfield network is mostly used for solving optimization problems, by mapping the optimization problem over a neural network. So that the network configurations correspond to possible solutions of the considered problem.

The dynamics of the network that present the output of the neurons in the Hopfield network are described by

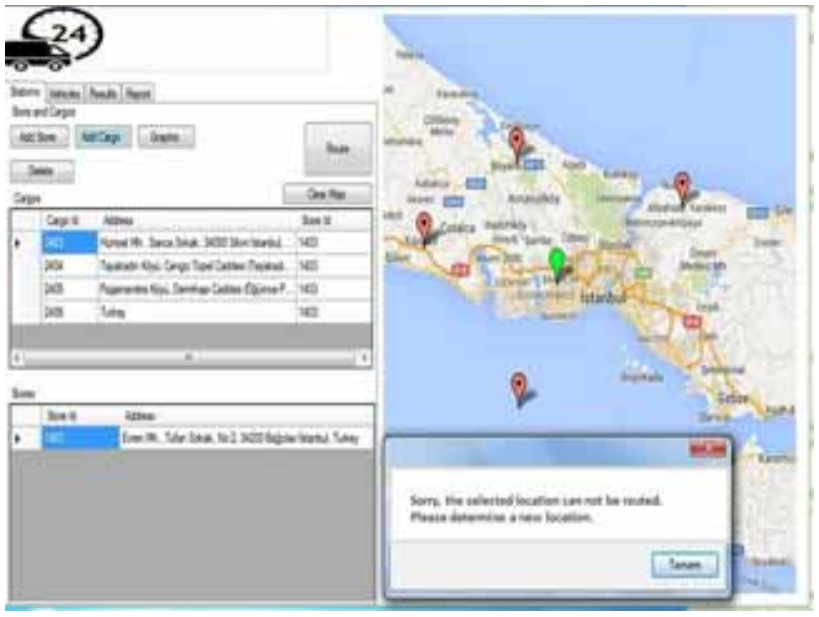

Figure 14. A warning message for a localization problem.

$$
\frac{\mathrm{d} u_{i}}{\mathrm{~d} t}=-\frac{u_{i}}{r}+\sum_{j=1}^{N} T_{i, j} V_{J}+I_{i}
$$

where $V=f\left(u_{i}\right)$ is output signal (vector) of neuron. Also, $u$, $T$, and $I$ are the state vector of neuron, connection strength matrix and bias of the $i$ th neuron, respectively; $r$ is the RC time constant; $u_{\mathbf{0}}$ is a constant.

The output function is given by

$$
V_{x i}=f\left(u_{x i}\right)=\frac{1}{2}\left(1+\tan h\left(\frac{u_{x i}}{u_{\mathbf{0}}}\right)\right) \text { for all } x, i
$$

The general energy function for the Hopfield network [22] is described by

$$
E=-\frac{1}{2} \sum_{i=1}^{N} \sum_{j=1}^{N} T_{\mathrm{i}, \mathrm{j}} V_{i} V_{j}-\sum_{i=1}^{N} V_{i} I_{i}
$$

where $N$ is the number of neurons, $T_{i j}$ represents the weight of interconnect between the input of the neuron $i$ and the output of the neuron $j$.

The energy equation [23] is given by

$$
\begin{aligned}
E= & \frac{A}{2} \sum_{x} \sum_{i} \sum_{j \neq i} V_{x i} V_{x j}+\frac{B}{2} \sum_{i} \sum_{x} \sum_{x \neq y} V_{x i} V_{y i} \\
& +\frac{C}{2}\left(\sum_{x} \sum_{i} V_{x i}-K\right)^{2} \\
& +\frac{D}{2} \sum_{x} \sum_{y \neq x} \sum_{i} d_{x y} V_{x i}\left(V_{y, i+1}+V_{y, i-1}\right)
\end{aligned}
$$

where $K$ is used as a parameter to adjust the neutral position of the amplifiers utilized to construct the network [23].

6.1a Tabu Search on Hopfield neural network comparison with the proposed structure: In optimization problems that are NPhard for heuristic approach, Hopfield method was also used. In 
Table 2. Tour length results of scenarios.

\begin{tabular}{lccc}
\hline & Minimum & Maximum & Average \\
\hline 16-city scenario & 2681 & 4228 & 3705 \\
$\begin{array}{c}\text { 17-city scenario (Tabu Search } \\
\text { on HopefieldNN scenario) }\end{array}$ & 3441 & 4985 & 4497 \\
\hline
\end{tabular}

traveling salesman problem, when gradient descent based Hopfield neural network is used as a method, it is hard to gain an optimal solution. Because of its gradient descent based structure and many local minima owning energy function. Thus, to gain better solutions, it's very important to escape from local minima. To improve the quality of the solutions together with the computation time, a tabu search into a Hopfield type neural network method is proposed for taking advantage of flexible and simple tabu search in [24] for TSP. With providing a proper energy function, Hopfield method can be utilized for many problems. But, there are two factors that affect the quality of the gained solutions and computation time. The first one is the energy function's multiple local minima. And the proportional rise of neurons to the square of the number of cities is the other vital factor. Tabu search is used to overcome both of these problems. The Hopfield network structure and energy function is presented together with the coefficients in [24]. Here, to find a better solution, tabu search discards local minima with enforcing limitation for utilization of short term memory of last solutions. Main idea and algorithm of this paper is used on VRP with its 17-city scenario for comparing the proposed 16-city scenario.

The proposed 16-city scenario is compared with the 17 city problem data which is gr-17 from TSPLIB [25]. Average tour length, minimum tour length and maximum tour lengths are considered. The proposed 16-city scenario produced better results than the compared 17-city scenario as presented in table 2 .

\subsection{Tabu search and SA comparisons with the proposed structures}

SA is a metaheuristic and it was introduced in [26]. It is an iterated local search algorithm that enables bad solutions to escape from local minima. To prevent from low quality solution, non-improving moves are probabilistically accepted in an attempt. With its simple logic, the cyclic order neighbourhoods can be utilized in many problems such as the VRP and the set covering problem $[27,28]$. SA is a hill climbing algorithm but it is slower than a simple hill climbing procedure thus it needs longer computation time. Also, in the search space, it can escape from local optima with its speciality [29].

An SA algorithm for the two-dimensional loading heterogeneous fleet vehicle routing problem (2L-HFVRP) is proposed with a heuristic local search in [30]. In VRP, the impact of triangle inequality violations on solution quality is presented considering SA, ant colony

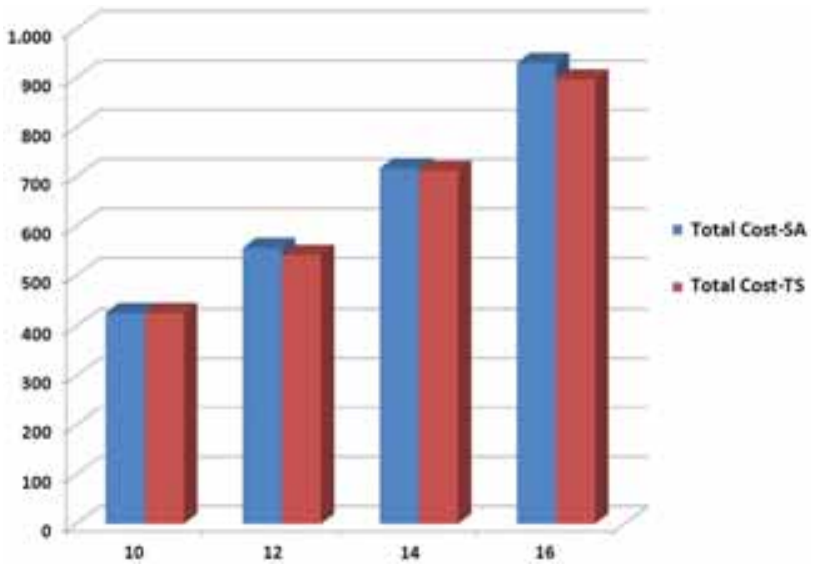

Figure 15. Total costs of tabu search and simulated annealing based 2-phase heuristic.

optimization and savings algorithm methods in the paper [31]. In [32], a hybrid approach is presented to minimize the total distance and costs for the third party reverse logistics provider (3PRLP). In the study, Sweep and Clarke-Wright savings algorithm is combined with SA algorithm. A combination of harmony search and SA metaheuristics is presented as a new integer programming model for transportation with cross-docking, direct shipment and milk run logistics in [33]. In [34], a vehicle routing problem with time windows (VRPTW) is considered together with deciding best routes and finding lowest travel cost. An SA approach is utilized in the presented novel mathematical model to overcome the problem.

Total cost in currency is compared for different number of customers in figure 15. The tabu search and SA algorithms are implemented on randomly generated scenarios with one depot and nearly 20 customers. In SA based 2-phase heuristic, the first phase includes the assigning of customers to the depots and in the second phase an SA algorithm for solving the single depot VRP is proposed. And collected results are compared. Our proposed tabu Search based scenarios are compared with the SA based scenario in [35]. The tabu Search gives better solution when compared to the SA based solution for total cost in currency. There is small gap between gained solutions of algorithms, and usually the gap between tabu search and SA solutions is equal to zero.

Tabu search and SA is known as similar in many ways. However, they differ in analysing the search space and achieving convergence. SA uses a cooling scheme but long and short term memory is used in tabu search $[36,37]$.

\section{Conclusion}

The system is designed to find the optimized routes for cargo firms while delivering the packets to the customers. Also, if it is demanded, it can be easily integrated to many 
sectors and areas. Because of the flexible structure of the program, instead of using the system only at cargo firms, the system can be used at school services or factory services that transports the students or workers in between their homes, schools or factories. It is very easy to utilize the program in different usage areas which are based on defining and finding optimized routes.

In cargo delivery systems, determining the routes, providing the delivery in a smooth manner and providing customer contentment are the most important factors. The proposed system will provide many advantages to related companies and corporations. The most important one of these advantages is finding and utilizing the shortest path, thus the corporations will gain great amounts of savings. Also, vehicle maintenance costs are decreased. Consequently, it is obvious that the system will provide big amounts of profit and acquisition. From the experimental results, it is shown that our algorithm outperforms the compared methods for the VRP problem.

Besides, t-test is conducted and p-values is calculated to decide if the presented algorithm's performance is good enough. It is realized that the presented tabu search algorithm's performance is better with $95 \%$ confidence at visited path in $\mathrm{km}$.

As a future work, in addition to decreasing costs, diminishing the emission rates of $\mathrm{Co} 2$ may be considered as a main study topic. Thus, the damage which is applied to nature may also be reduced.

\section{References}

[1] Dantzig G and Ramser J 1959 The truck dispatching problem. Manag. Sci. 6: 80-91

[2] Laporte G 1992 The vehicle routing problem: An overview of exact and approximate algorithms. Eur. J. Oper. Res. 59: 345-358

[3] Tan L, Lin F and Wang H 2015 Adaptive comprehensive learning bacterial foraging optimization and its application on vehicle routing problem with time windows. Neurocomputing 151(3): 1208-1215

[4] Barbucha D 2014 A cooperative population learning algorithm for vehicle routing problem with time windows. $\mathrm{Neu}$ rocomputing 146: $210-229$

[5] Ghilas V, Demir E and Woensel T M 2016 An adaptive large neighborhood search heuristic for the pickup and delivery problem with time windows and scheduled lines. Comput. Oper. Res. 72: 12-30

[6] Teymourian E, Kayvanfar V, Komaki G H M and Zandieh M 2016 Enhanced intelligent water drops and cuckoo search algorithms for solving the capacitated vehicle routing problem. Inf. Sci. 334-335: 354-378

[7] Setak M, Habibi M, Karimi H and Abedzadeh M 2015 A time-dependent vehicle routing problem in multigraph with FIFO property. J. Manuf. Syst. 35: 37-45

[8] Yu V F, Jewpanya P and Redi A A N P 2016 Open vehicle routing problem with cross-docking. Comput. Ind. Eng. 94: 6-17
[9] Expósito-Izquierdo C, Rossi A and Sevaux M 2016 A twolevel solution approach to solve the clustered capacitated vehicle routing problem. Comput. Ind. Eng. 91: 274-289

[10] Li J, Pardalos P M, Sun H, Pei J and Zhang Y 2015 Iterated local search embedded adaptive neighborhood selection approach for the multi-depot vehicle routing problem with simultaneous deliveries and pickups. Expert Syst. Appl. 42(7): 3551-3561

[11] Montoya-Torres Jairo R, Franco J L, Isaza S N, Jiménez H F and Herazo-Padilla N 2015 A literature review on the vehicle routing problem with multiple depots. Comput. Ind. Eng. 79: $115-129$

[12] Drexl M 2013 Applications of the vehicle routing problem with trailers and transshipments. Eur. J. Oper. Res. 227(2): 275-283

[13] Contardo C and Martinelli R 2014 A new exact algorithm for the multi-depot vehicle routing problem under capacity and route length constraints. Discrete Optim. 12: 129-146

[14] Kumar S N and Panneerselvam R 2012 A survey on the vehicle routing problem and its variants. Intell. Inf. Manag. 4: 66-74

[15] Glover F 1989 Tabu search - Part 1. ORSA J. Comput. 1(2): 190-206

[16] Glover F 1990 Tabu search - Part 2. ORSA J. Comput. 2(1): 4-32

[17] Piniganti L 2014 A survey of tabu search in combinatorial optimization thesis. University of Nevada, Las Vegas

[18] Froger A, Gendreau M, Mendoza J E, Pinson E and Rousseau L M 2016 Maintenance scheduling in the electricity industry: A literature review. Eur. J. Oper. Res. 251: 695-706

[19] Glover F 1990 Tabu search: A tutorial. Interfaces 20(1): 74-94

[20] Jia H, Li Y, Dong B and Ya H 2013 An improved tabu search approach to vehicle routing problem. Procedia-Social Behav. Sci. 96: 1208-1217

[21] Hopfield J J 1982 Neural networks and physical systems with emergent collective computational abilities. Proc. Natl. Acad. Sci. USA 79: 2554-2558

[22] Hopfield J J and Tank D W 1985 Neural computation of decisions in optimization problems. Biol. Cybern. SpringerVerlag 52: 141-152

[23] Kashmiri S 1991 The travellıng salesman problem and the Hopfield neural network. IEEE Proceedings of Southeastcon '91, Williamsburg, VA. 7-10(2): 940-943

[24] Konishi J, Shimba S, Toyama J, Kudo M and Shimbo M 1999 Tabu search for solving optimization problems on Hopfield neural networks. 1999 Third intematioml conference on knowledge-based intelligent lnformation engineeing systems, Adelaide, Australia

[25] TSPLIB. TSPLIB is a library of sample instances for the TSP. http://elib.zib.de/pub/mp-testdata/tsp/tsplib/tsplib.html. Visiting date: 20.11.2015

[26] Kirkpatrick S, Gelatt J and Vecchi M 1983 Optimization by simulated annealing. Science 220: 671-680

[27] Goodson J C 2010 Solution methodologies for vehicle routing problems with stochastic demand. $\mathrm{PhD}$ (Doctor of Philosophy) thesis, University of Iowa

[28] Stegers E 2009 A solution method for vehicle routing problems with time-dependent travel times. Master thesis, Delft University of Technology, Netherlands

[29] Hosny M I 2010 Investigating heuristic and meta-heuristic algorithms for solving pickup and delivery problems. $\mathrm{PhD}$ 
thesis, Cardiff University School of Computer Science \& Informatics

[30] Leung S C H, Zhang Z, Zhang D, Hua X, Lim M K 2013 A meta-heuristic algorithm for heterogeneous fleet vehicle routing problems with two-dimensional loading constraints. Eur. J. Oper. Res. 225(2): 199-210

[31] Fleming C L, Griffis S E and Bell J E 2013 The effects of triangle inequality on the vehicle routing problem. Eur. J. Oper. Res. 224(1): 1-7

[32] Sasikumar P, Haq A N and Baskar P 2010 A hybrid algorithm for the vehicle routing problem to third party reverse logistics provider. IEEE 8th International Conference on Supply Chain Management and Information Systems (SCMIS), HongKong, 1-8

[33] Hosseini S D, Shirazi M A and Karimi B 2014 Cross-docking and milk run logistics in a consolidation network: A hybrid of harmony search and simulated annealing approach. $J$. Manuf. Syst. 33(4): 567-577
[34] Tavakkoli-Moghaddam R, Gazanfari M, Alinaghian M, Salamatbakhsh A and Norouzi N 2011 A new mathematical model for a competitive vehicle routing problem with time windows solved by simulated annealing. J. Manuf. Syst. 30(2): 83-92

[35] Fard F A and Setak M 2011 Comparison between two algorithms for multi-depot vehicle routing problem with inventory transfer between depots in a three-echelon supply chain. Int. J. Comput. Appl. (0975-8887) 28(6): 39-45

[36] Takes F 2010 Applying Monte Carlo techniques to the capacitated vehicle routing problem. Master thesis, Leiden University, The Netherlands

[37] De A, Awasthi A and Tiwari M K 2015 Robust formulation for optimizing sustainable ship routing and scheduling problem. IFAC-PapersOnLine 48(3): 368-373 